\title{
No Association Between Ljungan Virus Seropositivity and the Beta-cell Damaging Process in the Finnish Type 1 Diabetes Prediction and Prevention Study Cohort
}

\section{Jääskeläinen, Anne J.}

2019-03

Jääskeläinen, A J , Nurminen , N , Kolehmainen , P , Smura , T , Tauriainen , S , Toppari , J , Ilonen , J , Veijola , R , Knip , M , Hyöty , H , Vapalahti , O \& Kallio-Kokko , H 2019 , ' No Association Between Ljungan Virus Seropositivity and the Beta-cell Damaging Process in the Finnish Type 1 Diabetes Prediction and Prevention Study Cohort ', Pediatric Infectious Disease Journal , vol. 38 , no. 3 , pp. 314-316 . https://doi.org/10.1097/INF.0000000000002201

http://hdl.handle.net/10138/326014

https://doi.org/10.1097/INF.0000000000002201

cc_by_nc

acceptedVersion

Downloaded from Helda, University of Helsinki institutional repository.

This is an electronic reprint of the original article.

This reprint may differ from the original in pagination and typographic detail.

Please cite the original version. 
The Pediatric Infectious Disease Journal Publish Ahead of Print

DOI: 10.1097/INF.0000000000002201

No Association Between Ljungan Virus Seropositivity and the Beta-Cell Damaging Process in the Finnish Type 1 Diabetes Prediction and Prevention Study Cohort Anne J. Jääskeläinen ${ }^{1}$, PhD, Noora Nurminen, ${ }^{2}$ MSc, Pekka Kolehmainen, ${ }^{1,3} \mathrm{PhD}$, Teemu Smura $^{1}, \mathrm{PhD}$, Sisko Tauriainen ${ }^{3}, \mathrm{PhD}$, Jorma Toppari ${ }^{4,5}, \mathrm{MD}, \mathrm{PhD}$, Jorma Ilonen ${ }^{6}, \mathrm{MD}, \mathrm{PhD}$, Riitta Veijola $^{7}$, MD, PhD, Mikael Knip ${ }^{8,9,10,11}$, MD, PhD, Heikki Hyöty ${ }^{2,12}$, MD, PhD, Olli Vapalahti ${ }^{1,13}, \mathrm{MD}, \mathrm{PhD}$, and Hannimari Kallio-Kokko, ${ }^{1} \mathrm{PhD}$

Corresponding author: Anne J. Jääskeläinen, Associate professor, $\mathrm{PhD}$, Hospital microbiologist, Helsinki University and Helsinki University Hospital, Department of Virology, P.O.B. 21 (Haartmaninkatu 3), FIN-00014 Univ. of Helsinki, Finland. Email: anne.jaaskelainen@ helsinki.fi, Mobile phone: +358 50 5420743, Office phone: +358 9 19126671, Fax: +358 919126491

Abbreviated title: No Association Between Ljungan Virus and Type 1 Diabetes Running head: Ljungan Virus and Type 1 Diabetes

${ }^{1}$ Helsinki Uniyersity and Helsinki University Hospital (HUSLAB), Department of Virology, Finland

${ }^{2}$ Department of Virology, School of Medicine, University of Tampere, Tampere, Finland ${ }^{3}$ Department of Virology, University of Turku, Turku, Finland

${ }^{4}$ Department of Physiology, Institute of Biomedicine, University of Turku, Turku, Finland ${ }^{5}$ Department of Pediatrics, Turku University Hospital, Turku, Finland ${ }^{6}$ Immunogenetics Laboratory, University of Turku and Turku University Hospital, Turku, Finland 
${ }^{7}$ Department of Pediatrics, PEDEGO Research Unit, Medical Research Center, Oulu

University Hospital and University of Oulu, Oulu, Finland

${ }^{8}$ Children's Hospital, University of Helsinki and Helsinki University Hospital, Helsinki,

Finland

${ }^{9}$ Research Programs Unit, Diabetes and Obesity, University of Helsinki, Helsinki, Finland

${ }^{10}$ Folkhälsan Research Center, Helsinki, Finland

${ }^{11}$ Tampere Center for Child Health Research, Tampere University Hospital, Tampere,

Finland

${ }^{12}$ Fimlab Laboratories, Pirkanmaa Hospital District, Tampere, Finland

${ }^{13}$ Faculty of Veterinary Medicine, Department of Veterinary Biosciences, University of Helsinki, Finland.

Funding: The study was financially supported by the HUSLAB (Helsinki University Hospital, Helsinki, Finland), Sigrid Juselius Foundation, the Academy of Finland, Reino Lahtikari Foundation and the Foundation for Diabetes Research in Finland. DIPP study has additionally been supported by grants from Juvenile Diabetes Research Foundation (JDRF) and the Competitive Research Funding of the Oulu, Tampere and Turku University Hospitals. Authors do not have any conflict of interest.

Acknowledgements We thank Cynthia Chi and Laura Paanila for excellent technical assistance and Lorna Culverwell for language editing. 


\section{Abstract \\ Background}

Ljungan virus (LV) has not confirmed to associate with any human disease, but a possible connection with type 1 diabetes (T1D) has been suggested. Ljungan virus (LV) is a rodent-borne picornavirus that induces a diabetes-like condition in rodents. Approximately $30 \%$ of adults and $60 \%$ of children are seropositive in Finland. The Finnish Type 1 Diabetes Prediction and Prevention (DIPP) study enabled the use of very well characterized sample panels from children seroconverted to positivity for multiple islet autoantibodies during their prospective observation from birth, in addition samples from age, sex, HLA and residence area matched control children.

\section{Methods}

We analyzed LV IgG seroprevalence in 102 case children (65 had also developed T1D), in addition to non-diabetic control children. LV and human parechovirus (HPeV) immunofluorescence assays were used to analyze LV and HPeV specific IgG from 102 plasma samples taken at the time of islet autoantibody appearance, and from 204 samples from the matched control children.

\section{Results}

Altogether $46.1 \%$ of the case and $50.7 \%$ of the control children were positive for LV IgG (odds ratio $0.8,95 \% \mathrm{CI} 0.47-1.36, \mathrm{p}=0.416$ ), and $67.6 \%$ vs. $79.8 \%$ were positive for $\mathrm{HPeV} \operatorname{IgG}$, respectively (odds ratio $0.49,0.27-0.9, \mathrm{p}=0.023$ ).

\section{Conclusions:}

Thus, no risk associations between $\mathrm{LV}$ or $\mathrm{HPeV}$-specific $\mathrm{IgG}$, and islet autoimmunity were observed. However, a trend for significantly higher prevalence of $\mathrm{HPeV}$ antibodies in control 
children $(\mathrm{p}=0.023)$ suggests a possible protective association of this virus with islet autoimmunity.

Keywords: Ljungan virus, LV, human parechovirus, HPeV, DIPP, diabetes, type 1 diabetes

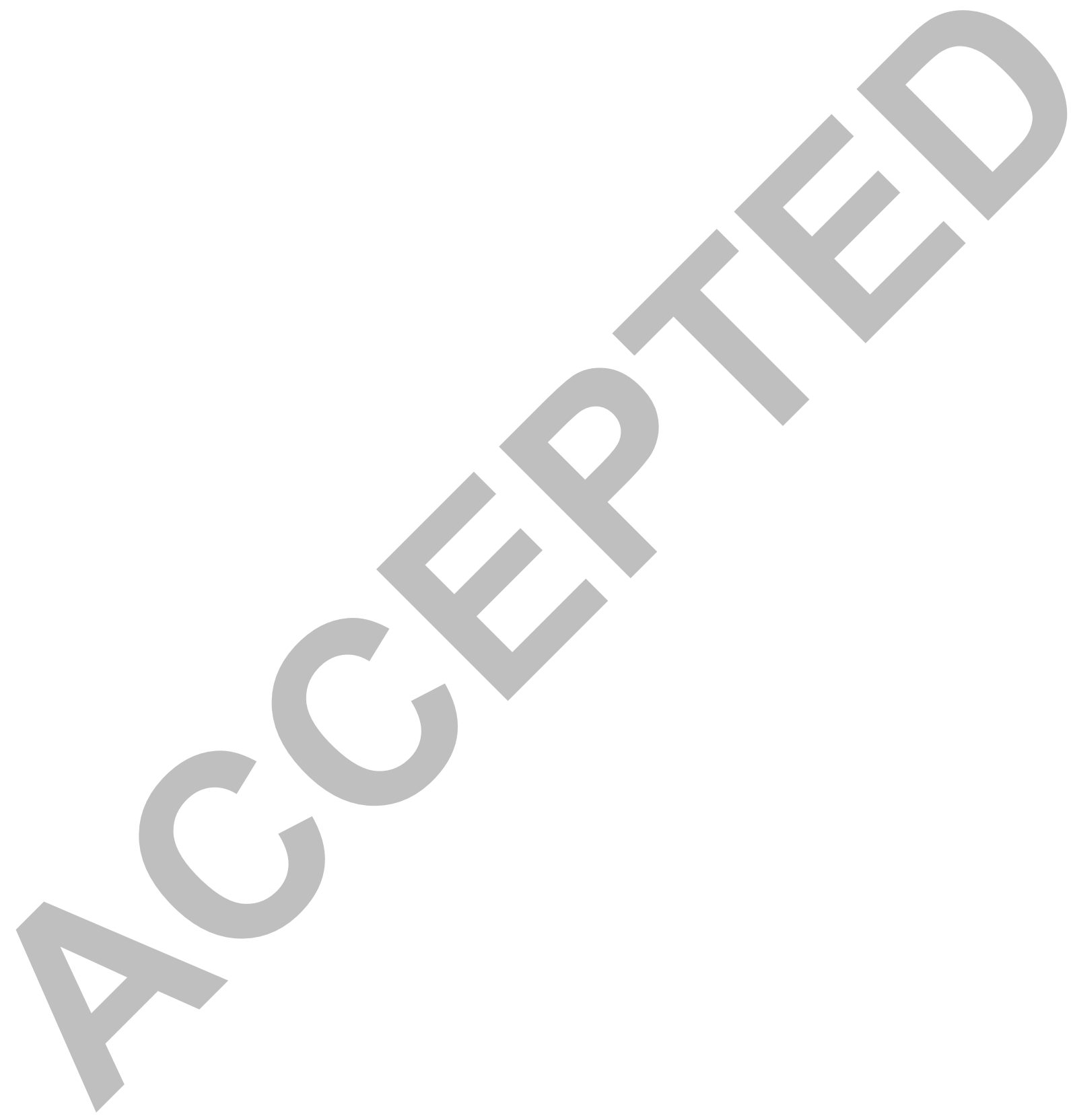




\section{Introduction}

Parechoviruses (Picornaviridae: Picornavirus) are small non-enveloped single-stranded RNA viruses. The genus Parechovirus comprises four species, and species of Parechovirus A [formerly Human parechovirus (HPeV)] and Parechovirus B [formerly Ljungan virus (LV)] consist of $16 \mathrm{HPeV}$ and $4 \mathrm{LV}$ types, (www.picornaviridae.com website, November 2017). LV is a rodent-borne virus (1) while HPeVs infect humans with symptoms including mild gastrointestinal and respiratory infections in children, and neonatal sepsis-like disease and encephalitis in infants (2).

The external triggers of human type 1 diabetes (T1D) have been the subject of extensive research, and certain virus infections, particularly enteroviruses have been implicated as the main candidates (3). Possible association of LV and diabetes has been studied using both nucleic acid detection methods and serological methods. In a MIDIA cohort study (4), LV RNA was not detected in stool samples collected from children in Norway. In Sweden, LV IgG antibodies were found to be associated with both positivity for HLA-DQB $1 * 0302$ (DQ8) allele and T1Dassociated islet autoantibodies $(5,6)$. In addition to $\mathrm{LV} \operatorname{IgG}$, the prevalence of $\mathrm{HPeV} \operatorname{IgG}$ was also increased in T1D patients with insulin autoantibodies and HLA-DQ8 when analyzed using multiplex immunoassay. However, the authors stated that cross-reactions could not be ruled out with this assay (6).

Our aim was to screen both LV- and HPeV-specific IgG antibodies from children who have seroconverted for multiple T1D-associated autoantibodies as a marker of an ongoing beta-cell damaging process and from their matched healthy control children in the DIPP study. This study design enabled the identification of infections which preceded the initiation of the beta-cell damaging process and could thus contribute to the initiation of this process. 


\section{Methods}

\section{Study Subjects}

All study subjects were participants in the DIPP study. The present study cohort included children recruited at the University Hospital in Tampere. Blood samples were drawn at the ages of $3,6,12,18$, and 24 months and once a year thereafter, and all follow-up sera were screened for T1D-associated autoantibodies [islet cell autoantibodies (ICA) as well as autoantibodies to insulin (IAA), glutamic acid decarboxylase 65 (GADA) and the protein tyrosine phosphataserelated IA-2 antigen (IA-2A)]. The DIPP study has been approved by the ethical committees of the participating university hospitals. A written informed consent was obtained from all participating families.

The following criteria were used to select children for the nested case-control study: The case children ( $\mathrm{N}=102,64 \%$ males) had turned positive for two or more of the T1D-associated autoantibodies mentioned above and remained autoantibody positive in all later follow-up samples. Of these, 65 have also progressed to clinical T1D. Two autoantibody negative and nondiabetic control subjects $(\mathrm{N}=203$, only one control child was available for one of the case children) were selected for each case child and matched for time of birth ( \pm 2 months), age at sample collection ( \pm 3 months), sex and T1D-associated HLA-DQ alleles. The first autoantibody positive sample of the case children and the corresponding sample from their matched control children were selected for virus antibody analyses (median age was 48 months and range 17-133 months at sample collection). The children were born in 1995-2009.

\section{Diabetes-Associated Autoantibodies and HLA Genotyping}

ICA was analyzed using indirect immunofluorescence assay (IFA) and IAA, GADA and IA-2A using radiolabel-binding assays (7). Genotypes associated with susceptibility to, or protection 
against, type 1 diabetes were identified by analyzing HLA-DQB1 alleles (DQB1*02, *03:01, $* 03: 02$, and *06:02/3) from cord blood of the children. The genotyping was based on lanthanide labeled oligonucleotide probe hybridization and time-resolved fluorometric detection (8). Children carrying the high-risk HLA-DQB1*02/DQB1*0302 genotype or the moderate-risk DQB1*0302/x genotype $(\mathrm{x} \neq \mathrm{DQB} 1 * 03: 02, * 06: 02$, or $* 06: 03)$ were invited for follow-up. The diagnosis of type 1 diabetes was based on the World Health Organization criteria.

\section{Virus antibodies}

LV- and HPeV-specific IgG was detected using LV (LV strain 145SLG; described in

Jääskeläinen et al. 2013) and HPeV IFAs. The HPeV IFA was set up using HPeV1 strain Harris and the method described in Jääskeläinen et al. (9), and was further evaluated by comparing the results with those obtained from $9 \mathrm{LV}$-antibody negative plasma samples using a $\mathrm{HPeV}$ microneutralization assay (MNT; 10). Two commercial hyperimmune antisera against HPeV1 and HPeV2 [VR-1063AS/HOTM, VR-1064AS/HO'TM; produced in horse, LGC Standards (ATCC), Teddington, United Kingdom] were used as controls.

\section{Statistical Analyses}

The prevalence of virus antibodies in both case and control groups was analyzed using conditional logistic regression and STATA 12.1 statistical software (StataCorp, College Station, TX, USA). A p value of less than 0.05 was set as indicator of statistical significance.

\section{Results}

In the comparison of HPeV IFA test with microneutralization assay, concordant results were obtained and HPeV IFA detected all HPeV1-6 types (8 positive samples). The titers for HPeV1-6 varied from 64 until 16384, and LV IFA was negative in all cases. One MNT negative sample was negative in both $\mathrm{HPeV}$ and LV IFA. In total, 49\% (150/305) of all DIPP children were 
seropositive for LV IgG and 76\% (231/305) HPeV IgG in IFA. Only one child was HPeV negative but LV positive. However, 84 children $(27.5 \%$; 84/505) were $\mathrm{HPeV}$ positive but LV negative (Table 1). The prevalence of LV antibodies did not differ between the case and control children (Table 1). This was also true when only those case children who progressed to clinical T1D and their controls were included. However, case children tended to be less frequently positive for $\mathrm{HPeV}$ antibodies than control children (Table 1). This trend was observed in both younger and older children and it was particularly clear among boys and in the subgroup of cases that had progressed to clinical T1D. Similar trend was further seen in children who developed IAA as the first appearing autoantibody and in those who seroconverted first to positivity for GADA (data not shown). The parallel presence of antibodies against LV and HPeV showed a significant correlation $(\chi 2=69.04, \mathrm{df}=1, \mathrm{p}<0.00001)$ in whole study cohort.

\section{Discussion}

LV infections have been linked to human T1D in some studies $(16,17)$ but prospective studies are lacking. In this study, both LV (49\%) and HPeV (76\%) antibodies were frequently detected. LV IFA was previously evaluated and no cross-reactivity was detected for HPeV1-2 and 4-6 (9). In the present study we tested the HPeV IFA using plasma samples positive for neutralizing antibodies (MNT) against HPeV1-6. All MNT positive samples were positive by HPeV IFA but negative by LV IFA.

LV seropositivity was not associated with the beta-cell damaging process as $46 \%$ of the case children, compared to $51 \%$ of their age, sex and HLA matched control children, were positive for LV antibodies ( $\mathrm{p}=0.416)$. This was also the case for HPeV IFA where $68 \%$ vs. $80 \%$ of the children analyzed were positive ( $\mathrm{p}=0.023$ ), respectively. Thus, the prevalence of $\mathrm{HPeV}$ antibodies was significantly lower in case children than in control children. The reason to this 
difference is not known. Overall, the control children were more HPeV seropositive than cases ( $p=0.023$ ), especially when the group of clinical TD1, boys and children over three years were examined ( $\mathrm{p}=0.007,0.009,0.029$, respectively; Table 2 ). The case children had no diabetesassociated metabolic changes, since the virus antibodies were analyzed in the early prediabetic phase when the children were still normoglycaemic. However, we cannot exclude the possibility that the function of the immune system is affected by the autoimmune process, leading to lower levels in $\mathrm{HPeV}$ antibodies. Alternatively, it is possible that $\mathrm{HPeV}$ infections could somehow protect against islet autoimmunity, possibly by stimulating the protective elements of the gut immune system. Accordingly, for enteroviruses, a link between infections with low-virulence virus strains and protection against T1D has been suggested (11). It should also be noted that maternal virus antibodies could not have biased these findings since all children were older than one year and maternal antibodies should have disappeared by that age. In Sweden, HLA-DQB*0302 and islet autoantibodies, particularly insulin autoantibodies, were suggested to be associated with LV and HPeV $\operatorname{IgG}$ antibodies (5, 6). In Norway and Finland, the children participating in the MIDIA and DIPP studies, respectively, were studied for parechovirus nucleic acids and/or antibodies by Tapia et al. (4), and Zhao et al. (12). No LV RNA positive feces in the MIDIA cohort (4) nor DIPP cohort (12). All children in the analyzed DIPP cohort samples have been DQB $1 * 0302$ positive based on earlier eligibility criteria and it has thus not been possible to explore the HLA association here.

This is a prospective study covering the time before the initiation of islet autoimmunity. This is the strength of this study, as well as, that the control children were carefully matched with to cases, and the LV and HPeV IgG IFAs were analyzed in parallel using validated methods. 
However, this study was carried out only in Finland, and this can be considered as a limitation to make any final conclusions.

The data also indicated that control children were statistically more positive for HPeV than case group, even when clinically T1D cases were analyzed. This is interesting finding, and raised the question do the HPeV antibodies somehow protect from T1D?

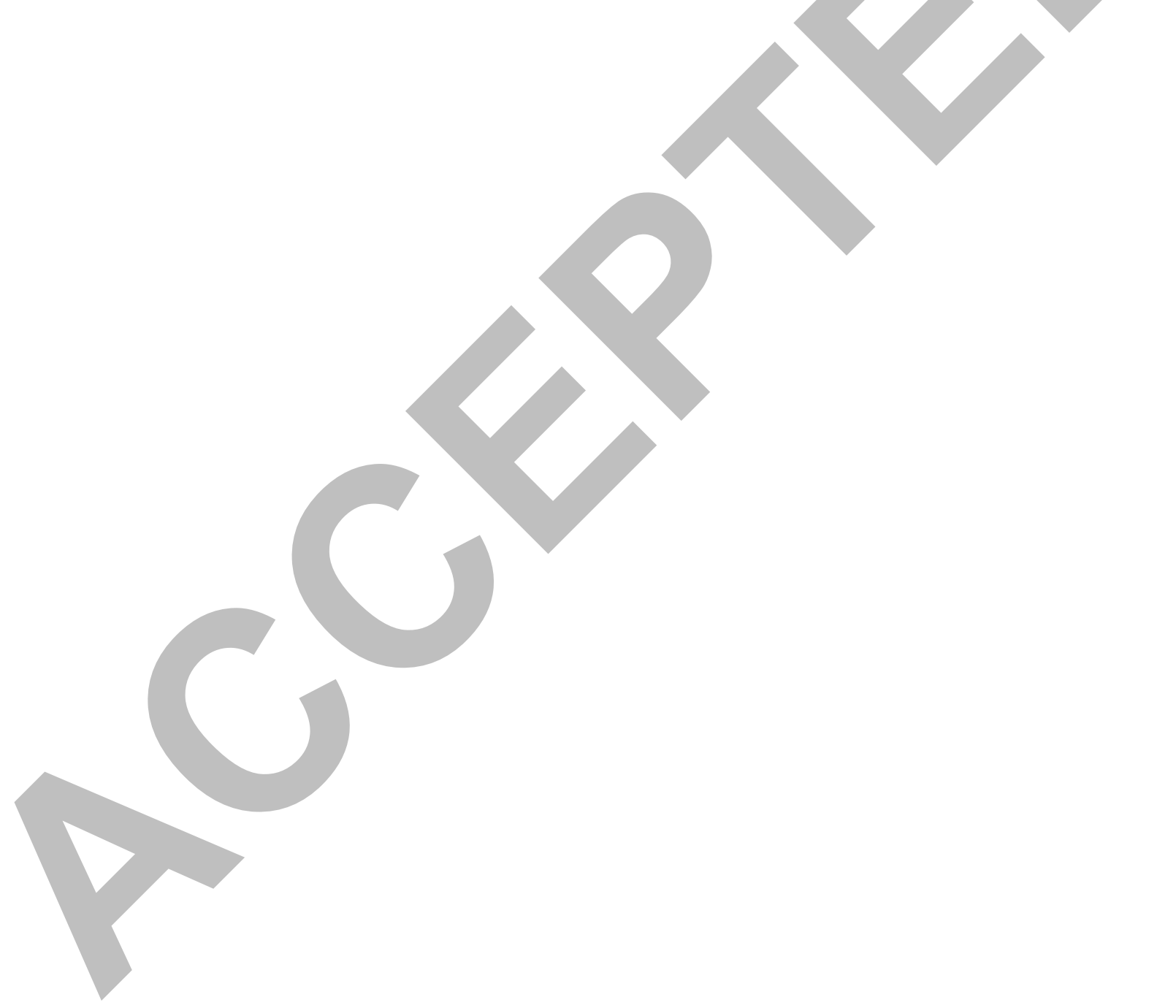




\section{References}

1. Niklasson B, Kinnunen L, Hornfeldt B, et al. A new picornavirus isolated from bank voles (Clethrionomys glareolus). Virology 1999; 255:86-93

2. Verboon-Maciolek MA, Krediet TG, Gerards LJ, et al. Severe neonatal parechovirus infection and similarity with enterovirus infection. Pediatr Infect Dis J. 2008; 27:241-5.

3. Nurminen N, Oikarinen S, Hyöty H. Virus infections as potential targets of preventive treatments for type 1 diabetes. Rev Diabet Stud Winter 2012; 9:260-271

4. Tapia G, Cinek O, Rasmussen T, et al. No_Ljungan_virus RNA in stool samples from the Norwegian environmental triggers of type 1 diabetes (MIDIA) cohort study.

Diabetes Care 2010; 33:1069-1071

5. Nilsson AL, Vaziri-Sani F, Andersson C, et al. Relationship between_Ljungan_virus antibodies, HLA-DQ8, and insulin autoantibodies in newly diagnosed type 1 diabetes children. Viral Immunol 2013; 26:207-215

6. Nilsson AL, Vaziri-Sani F, Broberg P, et al. Serological evaluation of possible exposure to Ljungan virus and related parechovirus in autoimmune (type 1) diabetes in children. $J$ Med Virol. 2015; 87:1130-1140.

7. Knip M, Virtanen SM, Seppä K, et al.; Finnish TRIGR Study Group. Dietary intervention in infancy and later signs of beta-cell autoimmunity. N Engl J Med 2010; 363:1900-1908

8. Sjöroos M, Iitiä A, Ilonen J, et al. Triple-label hybridization assay for type-1 diabetesrelated HLA alleles. Biotechniques. 1995; 18:870-7.

9. Jạäskeläinen AJ, Kolehmainen P, Voutilainen L, et al. Evidence of Ljungan virus specific antibodies in humans and rodents, Finland. J Med Virol 2013; 85:2001-2008 
10. Kolehmainen P, Koskiniemi M, Oikarinen S, et al. Human parechovirus and the risk of type 1 diabetes. J Med Virol. 2013; 85:1619-1623

11. Christen U, von Herrath MG. Do viral infections protect from or enhance type 1 diabetes and how can we tell the difference? Cell Mol Immunol 2011; 8:193-198

12. Zhao G, Vatanen T, Droit L, et al. Intestinal virome changes precede autoimmunity in type I diabetes-susceptible children. Proc Natl Acad Sci U S A. 2017; 114:E6166-E6175 
Table 1. Prevalence of LV and HPeV antibodies in children with $\beta$-cell autoimmunity (case children) and autoantibody-negative control children.

\begin{tabular}{|c|c|c|c|c|c|c|}
\hline \multirow[t]{2}{*}{ Group ( $N=$ in total) } & Seroprevalence of & Cases $^{1}$ & Controls $^{2}$ & OR & $95 \% \mathrm{Cl}$ & p- \\
\hline & LV and HPeV & N (\%) & N (\%) & & & value $^{3}$ \\
\hline \multirow{5}{*}{$\begin{array}{l}\text { Summary of all } \\
\text { (Cases } N=102 \text {, Controls } N=203 \text {; }\end{array}$} & LV & $47(46.1)$ & $103(50.7)$ & 0.8 & $0.47,1.36$ & 0.416 \\
\hline & $\mathrm{HPeV}$ & $69(67.6)$ & $162(79.8)$ & 0.49 & $0.27,0.90$ & 0.023 \\
\hline & LV and HPeV & $45(44.1)$ & $102(50.2)$ & 0.77 & $0.45,1.33$ & 0.354 \\
\hline & LV pos, HPeV neg & & $1(0.5)$ & NA & NA & NA \\
\hline & HpeV pos, LV neg & $(2$ & $60(30.5)$ & 0.67 & $0.36-1.23$ & 0.196 \\
\hline \multirow{2}{*}{$\begin{array}{ll}\text { Divided by sex } & \text { Boys } \\
\text { (Cases } N=102, & (N=192)\end{array}$} & LV & $27(42.2)$ & $68(53.1)$ & 0.56 & $0.28,1.12$ & 0.102 \\
\hline & $\mathrm{HPeV}$ & (65.1) & $104(81.2)$ & 0.35 & $0.16,0.77$ & 0.009 \\
\hline \multirow[t]{2}{*}{ All $N=305$ ) } & LV & $20(52.6)$ & 35 (46.7) & 1.35 & $0.59,3.12$ & 0.481 \\
\hline & $\mathrm{HPeV}$ & $29(78.4)$ & $59(79.7)$ & 0.91 & $0.31,2.62$ & 0.855 \\
\hline \multirow{2}{*}{ Divided by age } & LV & $16(47.1)$ & $31(45.6)$ & 1.09 & $0.41,2.89$ & 0.868 \\
\hline & HPeV & $22(64.7)$ & $49(73.1)$ & 0.63 & $0.22,1.77$ & 0.380 \\
\hline \multirow[t]{2}{*}{ Controls N=203; } & LV & $31(45.6)$ & $72(53.3)$ & 0.71 & $0.38,1.33$ & 0.283 \\
\hline & HPeV & $48(72.7)$ & $114(84.4)$ & 0.43 & $0.20,0.92$ & 0.029 \\
\hline
\end{tabular}


**Cases progressed to T1D

LV

$33(50.8) \quad 66(51.2) \quad 1.00 \quad 0.51,1.95 \quad 1.000$

( $N=65$ cases, their child

matched controls $N=129$ )

LV, Ljungan virus; HPeV, human parechovirus; T1D, type 1 diabetes; NA, not applicable;

${ }^{1}$ in total 102 children, gender: 64 males/38 females

${ }^{2}$ in total 203 children, gender: 128 males/75 females

${ }^{3}$ p-value $<0.05$ considered significant

** In total, 65 individual cases progressed to clinical T1D. None of the matched control children presented with T1D ( $\mathrm{n}=129$; one TD1 case had only one control child). 